\title{
Capgras syndrome and intellectual disability in Down Syndrome: A case report
}

\author{
Burcu Akın Sarı1 \\ ${ }^{1}$ Assis. Prof., Baskent University Faculty of Medicine, Department of Child and Adolescent Psychiatry, Ankara, Turkey \\ https://orcid.org/0000-0003-2106-7928
}

\section{TO THE EDITOR}

Down syndrome is a genetic disorder that result from trisomy 21. Delay in cognitive development (frequently mild to moderate intellectual disability is seen), adaptive behavior problems, and increased risk of depression and Alzheimer's disease are seen with this syndrome (1). For ages under 20 disruptive behaviors, anxiety disorders and repetitive behaviors are very commonly seen (2).

Capgras syndrome is a delusional misidentification syndrome, first described by Capgras and ReboulLachaux in 1923 (3). In this syndrome person believes that identical doubles have replaced significant people in his/her life (4). Prevalence rate of Capgras syndrome is unknown but in some studies $14 \%$ of rate is reported. The recent studies indicated cerebral dysfunction as an etiological factor of this syndrome (4). Capgras syndrome is associated with some organic disorders but not Down syndrome as far as we know (5).

In this case report, the patient with Down syndrome who also has moderate intellectual disability and Capgras syndrome is reported. Informed consent was taken from his parents. In literature the coexistence of these syndromes has not been reported as far as we know. The possible explanations of the coexistence of these syndromes are discussed.

\section{CASE REPORT}

A 14-year-old male was referred for special educa- tional treatment report to the department. Down syndrome had been diagnosed after birth. He had one sibling, a brother. There was no family history of any mental health disorder. He had special education since 3 -year-old. He walked at 2 years of age. He started to speak at 18 months of age. He had developmental delay at every developmental features. He had not got any cardiac, endocrinological or neurological deficiency. In his physical examination morbid obesity was detected. In mental examination his cognitive ability was retarded, his literacy ability was at first grade level. He was able to do simple math operations. There was no symptom of perception disorder. Two years later he came to examination again with his parents. In this examination cognitive abilities were still retarded and he was thinking as if his parents were not his real parents. They were somebody else but not the parents and he did not clarify why they look after him. Agitation, aggression were not seen but he said he was sorry because of that situation. His parents refused to use medication or any other treatment. Two years later he referred again, his beliefs about his parents were still existing. There were no other symptoms or his symptoms did not cause aggressive behaviors. No hallucination was detected in these examinations.

His cranial magnetic resonance investigation and electroencephalography findings were normal. He was unable to complete the Wechsler intelligence scale for children-revised.

\section{DISCUSSION}

This is the first case report with coexistence of 
Down syndrome and Capgras syndrome as far as we know. In literature Fregoli syndrome and Down syndrome coexistence was discussed. They suggested that this symptomatology can be explained in psychodynamic and developmental terms (6). Studies about Capgras and learning difficulties are rare and they suggested that this coexistence is due to psychodynamic etiology and right hemisphere dysfunction $(7,8)$. This case has moderate intellectual disability and he has normal neuroimaging results. Therefore we supposed that psychodynamic and neurological deficits do not explain the case's etiology. On the other hand Alzheimer disease is associated with Capgras syndrome and also Alzheimer disease is associated with Down syndrome (9). We evaluated that the cerebral dysfunction might be the etiological explanation however his MRI findings were normal even though he has Capgras syndrome. Therefore we suggested that this coexistence may be due to genetic features. Faber and Abrams also reported a case with Klinefelter's syndrome and they also suggested that chromosomal anomaly could alter brain functions as etiology of idiopathic schizophrenia (10).

The parents of this case refuse the treatment. If we can treat him, we can observe the medication or therapy results of this coexistence. But the patient's symptoms does not seem to limit his functions. He was just feeling sorry about this situation and depression criteria did not been met according to DSM-5. Hurley suggested that in Down syndrome, psychotic symptoms are not mood congruent (11). This may be the reason for the absence of aggression in this patient.

In conclusion, based on this case report, it can be said that Down syndrome and Capgras syndrome may be two diseases with common genetic origins. Further genetic studies are needed.

Informed consent form was taken from the patient's parents.

\section{Conflict of interest}

The author has no conflict of interest to declare.

\section{Funding}

There was no funding for this work

Correspondence address: Assoc. Prof., Burcu Akın Sarı, Baskent University Faculty of Medicine, Department of Child and Adolescent Psychiatry Ankara, Turkey carpediemburcu@yahoo.com

\section{REFERENCES}

1. Chapman RS, Hesketh LJ. Behavioral phenotype of individuals with Down syndrome. Ment Retard Dev Disabil Res Rev 2000;6(2):84-95. doi: 10.1002/1098-2779(2000)6:2<84::AIDMRDD2>3.0.CO;2-P.

2. Dykens EM, Shah B, Sagun J, Beck T, King BH. Maladaptive behaviour in children and adolescents with Down's syndrome. J Intellect Disabil Res 2002;46(Pt 6):484-92.

3. Capgras J, Reboul-Lachaux J. Lillusion des "sosies" dans un delire systematize chronique. Bull Soc Clin Ment 1923;11:6-16. https://doi.org/10.1177/0957154X9400501709

4. Barrelle A, Luauté JP. Capgras Syndrome and Other Delusional Misidentification Syndromes. Front Neurol Neurosci. 2018;42: 35-43. doi: 10.1159/000475680.

5. Pandis C, Agrawal N, Poole N. Capgras' delusion: a systematic review of 255 published cases. Psychopathology 2019:52:161173. doi: $10.1159 / 0005000474$

6. Collacott RA, Napier M. Erotomania and Fregoli-like started in Down's syndrome:dynamic and developmental aspects. J Ment Defic Res 1991;35:481-6. doi:10.1111/j.13652788.1991.tb00431.x
7. Mann J, Foreman DM. Homo-erotomania for a delusional parent: erotomania with Capgras and Fregoli syndromes in a young male with learning difficulties. J Intellect Disabil Res 1996:40:275-8.

8. Pachana NA, Drake E, van Gorp WG, Sorensen D. Similarities of neuropsychological presentation in two cases of Capgras Syndrome with comorbid depression New Zeal J Psychol 1999;28:55.

9. Edelstyn NM, Oyebode F. A review of the phenomenology and cognitive neuropsychological origins of the Capgras syndrome. Int $\mathbf{J}$ Geriatr Psychiatry 1999;14: 48-59. doi:10.1002/(SICI)1099-1166(199901)14:1<48::AIDGPS891>3.0.CO;2-0

10. Faber R, Abrams R. Schizophrenia in a 47,XYY male. Brit. J Psychiatr 1975;127: 401-3.

11. Hurley AD. Delusions and hallucinations in Down syndrome: literature review and comparison with Non-Down syndrome patients. Ment Health Asp Dev Disabil 2003;6:135-146 